\title{
KULEUVEN
}

\section{A smooth nonparametric, multivariate, mixed-data location-scale test}

Racine J, Van Keilegom I. 


\title{
A SMOOTH NONPARAMETRIC, MULTIVARIATE, MIXED-DATA LOCATION-SCALE TEST
}

\author{
JEFFREY S. RACINE \\ Department of Economics and Graduate Program in Statistics, McMaster University, \\ racinej@mcmaster.ca; Info-Metrics Institute, American University; Rimini Center for Economic \\ Analysis; Center for Research in Econometric Analysis of TimE Series (CREATES), Aarhus \\ University.
}

\section{INGRID VAN KEILEGOM}

ORSTAT, KU Leuven, Naamsestraat 69, B-3000 Leuven, Belgium.

\begin{abstract}
A number of tests have been proposed for assessing the location-scale assumption that is often invoked by practitioners. Existing approaches include Kolmogorov-Smirnov and Cramérvon-Mises statistics that each involve measures of divergence between unknown joint distribution functions and products of marginal distributions. In practice, the unknown distribution functions embedded in these statistics are typically approximated using non-smooth empirical distribution functions. In a recent article, Li, Li \& Racine (2017) establish the benefits of smoothing the empirical distribution function for inference, though their theoretical results are limited to the case where the covariates are observed and the distributions unobserved, while in the current setting some covariates and their distributions are unobserved (i.e., the test relies on population error terms from a location-scale model) which necessarily involves a separate theoretical approach. We demonstrate how replacing the non-smooth distributions of unobservables with their kernelsmoothed sample counterparts can lead to substantial power improvements, and extend existing approaches to the smooth multivariate and mixed continuous and discrete data setting in the presence of unobservables. Theoretical underpinnings are provided, Monte Carlo simulations are undertaken to assess finite-sample performance, and illustrative applications are provided.
\end{abstract}

\section{INTRODUCTION}

Assuming independence of the predictors and error in a location-scale regression model is a common assumption. The independence assumption is for instance needed for certain bootstrap

Date: December 21, 2018.

1991 Mathematics Subject Classification. C14 Semiparametric and Nonparametric Methods.

Key words and phrases. Kernel Smoothing; Kolmogorov-Smirnov; Inference.

Racine would like to gratefully acknowledge support from the Natural Sciences and Engineering Research Council of Canada (NSERC:www.nserc.ca), the Social Sciences and Humanities Research Council of Canada (SSHRC:www.sshrc.ca), and the Shared Hierarchical Academic Research Computing Network (SHARCNET:www.sharcnet.ca). I. Van Keilegom acknowledges financial support from the European Research Council (20162021, Horizon 2020 / ERC grant agreement No. 694409). 
procedures (see Neumeyer (2008), Neumeyer (2009), Neumeyer \& Van Keilegom (2018)). There is also an extensive literature on testing procedures that use the independence between the error and the covariates, and that are based on a comparison between a nonparametric estimator of the error distribution and an estimator under the null hypothesis. We refer for instance to Van Keilegom, González-Manteiga \& Sánchez-Sellero (2008) and Dette, Neumeyer \& Van Keilegom (2007) for goodness-of-fit tests for the parametric form of the regression and the variance function, respectively, to Pardo-Fernández, Van Keilegom \& González-Manteiga (2007) for tests for the equality of regression curves, and to Escanciano, Pardo-Fernández \& Van Keilegom (2018) for distribution-free tests in this context. A testing procedure for the location-scale structure having high power would be particularly appealing.

A variety of tests have been proposed for assessing the appropriateness of the location-scale assumption that is often invoked in applied settings; see by way of illustration Akritas \& Van Keilegom (2001) and Racine \& Li (2017), who adopt the location-scale framework, and see Einmahl \& Van Keilegom (2008), Birke, Neumeyer \& Volgushev (2017) and Neumeyer, Noh \& Van Keilegom (2016) for various approaches that have been proposed to test the location-scale assumption in a range of settings. These approaches employ test statistics that are based on conditional mean models, in particular, the difference between the joint distribution of the predictor and error and the product of the marginal distributions of the predictor and error, and include the KolmogorovSmirnov (Kolmogorov 1933, Smirnov 1948), Cramér-von-Mises (Cramér 1928, von Mises 1928) and Anderson-Darling (Anderson \& Darling 1952) statistics, among others. In this literature, the unknown joint and marginal distributions are estimated using the respective non-smooth empirical distribution functions (EDFs). However, it turns out that substantial power gains can be realized by replacing the non-smooth EDFs with their kernel-smoothed counterparts. We demonstrate that we retain all of the desirable features of this testing framework yet can realize substantial improvements to existing procedures from the vantage point of finite-sample power without impacting size. Though we consider inference for location-scale models, the results contained herein are of broad applicability and ought to appeal to a wide audience, particularly practitioners concerned with power properties associated with this popular class of test statistics. 
The remainder of this paper proceeds as follows: Section 2 outlines the location-scale framework and the proposed smooth testing procedure; Section 3 and Section 4 present the theoretical underpinnings of the proposed approach; Section 5 presents simulation evidence that demonstrates power gains achievable by a fully data-driven implementation of the proposed approach; Section 6 considers an illustrative application, while Section 7 presents some concluding remarks. The proofs of the main results are given in Appendix A, while detailed tables outlining power gains are presented in Appendix B and C.

\section{Methodology}

Consider a smooth location-scale model of the form

$$
Y=\mu(X)+\sigma(X) \epsilon
$$

where $\mu(\cdot)$ and $\sigma(\cdot) \geq 0$ are unknown smooth location and scale functions, $X$ is a vector of predictors, and $\epsilon$ has zero mean, unit variance, and is otherwise an unknown error process with distribution $F_{\epsilon}$ that is independent of $X$. We observe $n$ independent copies of $\left(X^{\mathrm{T}}, Y\right)$, denoted by $\left(X_{1}^{\mathrm{T}}, Y_{1}\right), \ldots,\left(X_{n}^{\mathrm{T}}, Y_{n}\right)$.

The location-scale assumption is often invoked as it confers a number of useful properties on the resulting estimator, including i) simpler asymptotic properties than its unstructured counterpart, ${ }^{1}$ ii) the ability to nonparametrically estimate the error distribution at a $\sqrt{n}$-rate (Akritas \& Van Keilegom 2001, Escanciano \& Jacho-Chávez 2012), and iii) more efficient estimation of the conditional distribution of $Y$ given $X$ than its unstructured counterpart.

Even though the independence of the predictors $X$ and error $\epsilon$ is a common assumption (see e.g., Akritas \& Van Keilegom (2001) and Racine \& Li (2017)), particularly in Econometrics, it might be too strong, hence a testing procedure having high power is particularly appealing. For what follows, we define $F_{X}(x)=P(X \leq x), F_{\epsilon}(t)=P(\epsilon \leq t)$, and $F_{X, \epsilon}(x, t)=P(X \leq x, \epsilon \leq t)$, and we let

$$
H_{0}: X \text { and } \epsilon \text { are independent. }
$$

\footnotetext{
${ }^{1} \mathrm{By}$ 'unstructured' we mean a model of the form $Y_{i}=\mu\left(X_{i}\right)+\epsilon_{i}$ with $E\left(\epsilon_{i} \mid X_{i}\right)=0$.
} 
Consider by way of illustration the test of Einmahl \& Van Keilegom (2008) which can be used to assess the adequacy of the location-scale assumption. In essence, Einmahl \& Van Keilegom (2008) test for independence between the predictors $X$ and error $\epsilon$ in the location-scale model $Y=\mu(X)+\sigma(X) \epsilon$. Given kernel estimates of $\mu(x)=E(Y \mid X=x)$ and $\sigma^{2}(x)=V(Y \mid X=x)$, denoted $\hat{\mu}(x)$ and $\hat{\sigma}^{2}(x)$, one tests for independence of $X_{i}$ and $\hat{\epsilon}_{i}=\left(Y_{i}-\hat{\mu}\left(X_{i}\right)\right) / \hat{\sigma}\left(X_{i}\right)$ using, for instance, a Kolmogorov-Smirnov test statistic of the form

$$
T_{K S}=\sqrt{n} \sup _{x, t}\left|\hat{F}_{X, \hat{\epsilon}}(x, t)-\hat{F}_{X}(x) \hat{F}_{\hat{\epsilon}}(t)\right|
$$

where $\hat{F}_{X, \hat{\epsilon}}(x, t), \hat{F}_{X}(x)$ and $\hat{F}_{\hat{\epsilon}}(t)$ are the respective EDFs. The empirical support, i.e., the $X_{i}$ and $\hat{\epsilon}_{i}$, are used when computing the supremum in applied settings. Due to the inadequacy of using the asymptotic distribution of $T_{K S}$ for inference, a simple bootstrap procedure is used instead to obtain the null distribution from which nonparametric $P$-values can readily be obtained. This procedure can be easily modified to test for the validity of a homoskedastic model $Y_{i}=\mu\left(X_{i}\right)+\epsilon_{i}$ with $\epsilon_{i}$ being independent of $X_{i}$, which is also a common assumption in Econometrics, or to test the validity of a transformation model of the form $\Delta\left(Y_{i}\right)=\mu\left(X_{i}\right)+\sigma\left(X_{i}\right) \epsilon_{i}$ as outlined in Neumeyer et al. (2016), where $\Delta(\cdot)$ is some parametric monotone transformation. See Neumeyer (2008) who demonstrate consistency of the bootstrap in the kernel-smoothed case, while Neumeyer \& Van Keilegom (2018) address the open question of whether a classical non-smooth residual bootstrap is asymptotically valid in this context, and show that the non-smooth residual bootstrap is consistent.

We note in passing that the Cramér-von-Mises statistic, which is also popular in applied settings, is given by

$$
T_{C M}=n \iint\left(\hat{F}_{X, \hat{\epsilon}}(x, t)-\hat{F}_{X}(x) \hat{F}_{\hat{\epsilon}}(t)\right)^{2} d \hat{F}_{X}(x) d \hat{F}_{\hat{\epsilon}}(t)
$$

We propose replacing the EDFs in these statistics with their kernel-smoothed counterparts and the unknown error term with its kernel estimate using an approach similar to Li et al. (2017) that is briefly described below. One major difference between the results established here and those in Li et al. (2017) is that, here, some covariates and distributions involve unobserved error terms that need to be estimated by $\hat{\epsilon}_{i}=\left(Y_{i}-\hat{\mu}\left(X_{i}\right)\right) / \hat{\sigma}\left(X_{i}\right)$, which results in markedly different asymptotics and finite sample performance from that reported in Li et al. (2017). Like Li et al. (2017), our approach is multivariate in nature and allows for mixed datatypes, but the presence 
of unobservables leads to results that to the best of our knowledge have not been exploited in the literature. Related work includes Conover (1999, pp. 396-406) who considers a smooth two-sample Kolmogorov-Smirnov test, ${ }^{2}$ Bowman, Hall \& Prvan (1998) who consider bandwidth selection for univariate kernel smoothed CDFs and Wang et al. (2013) who consider a plug-in bandwidth procedure for smooth univariate kernel smoothed CDFs with a focus on the construction of simultaneous confidence bands.

2.1. Kernel Estimation of $F_{X}(x), F_{\epsilon}(t)$, and $F_{X, \epsilon}(x, t)$ with Mixed Data. Though Einmahl \& Van Keilegom (2008) and others restrict attention to the scalar predictor case, in applied settings one would expect to encounter multivariate predictors that, in addition, might consist of both discrete and continuous datatypes. Though the Kolmogorov-Smirnov and Cramér-von-Mises statistics were developed under the assumption that the random variables possessed continuous distributions $F(\cdot)$ and have been extended to instead admit discrete distributions (Conover 1972, Gleser 1985, Choulakian, Lockhart \& Stephens 1994, Lockhart, Spinelli \& Stephens 2007), to the best of our knowledge they are unable to handle the multivariate mix of continuous and discrete data often found in regression settings. Our approach tackles this shortcoming by leveraging recent work on nonparametric kernel estimation of distributions involving a mix of discrete and continuous variables.

Li et al. (2017) propose an estimator of a joint distribution function defined over a mix of observed continuous and discrete random variables, which we will explain by means of the vector of covariates $X$. We suppose that $X_{j}(j=1, \ldots, n)$ is a $(q+r)$-dimensional vector of covariates, consisting of $q$ continuous covariates denoted by $X_{j}^{c}=\left(X_{j 1}^{c}, \ldots, X_{j q}^{c}\right)$ and $r$ (ordered) discrete covariates denoted by $X_{j}^{d}=\left(X_{j 1}^{d}, \ldots, X_{j r}^{d}\right)$. Likewise, $X$ consists of a $q$-dimensional vector of continuous covariates $X_{1}^{c}, \ldots, X_{q}^{c}$ and an $r$-dimensional vector of discrete covariates $X_{1}^{d}, \ldots, X_{r}^{d}$. The support of $X$ is denoted by $R_{X}=R_{X^{c}} \times R_{X^{d}}$, where $R_{X^{c}}$ is supposed to be a compact subset of $\mathcal{R}^{q}$. We consider smooth kernel-based estimators of $F_{X}(x)=P(X \leq x)=P\left(X^{c} \leq x^{c}, X^{d} \leq x^{d}\right)$ (where inequalities should be understood componentwise).

\footnotetext{
${ }^{2}$ This approach compares two kernel smoothed univariate distributions; see the function KS.test in the R package Qiu (2014) which implements this procedure using Wang, Cheng \& Yang's (2013) plug-in bandwidth and uses the asymptotic distribution for critical values which is known to be problematic.
} 
We consider discrete variables distributed over a finite grid, and without loss of generality assume that $X_{j s}^{d}$ takes values in $\left\{0,1, \ldots, c_{s}-1\right\}(s=1, \ldots, r)$, where $c_{s} \geq 2$ is a positive integer. Let $\lambda_{s}$ denote the bandwidth for the $s$-th discrete variable. We use the kernel function $l\left(x_{s}^{d}, X_{j s}^{d}, \lambda_{s}\right)=$ $\eta_{s} \sum_{z_{s}^{d} \leq x_{s}^{d}} \lambda_{s}^{\left|X_{j s}^{d}-z_{s}^{d}\right|}$, with $\lambda_{s}^{0}=1,0^{0}=1, \lambda_{s} \in[0,1]$, and $\eta_{s}$ a normalizing factor such that $l\left(c_{s}-\right.$ $\left.1, c_{s}-1, \lambda_{s}\right)=1$. Write the product (discrete variable) cumulative kernel function as $L_{\lambda}\left(x^{d}, X_{j}^{d}\right)=$ $\prod_{s=1}^{r} l\left(x_{s}^{d}, X_{j s}^{d}, \lambda_{s}\right)$.

Let $h_{s}$ be the bandwidth associated with $X_{s}^{c}(s=1, \ldots, q)$. The product cumulative kernel function used for the continuous variables is given by $K_{h}\left(x^{c}, X_{j}^{c}\right)=\prod_{s=1}^{q} \int_{-\infty}^{x_{s}^{c}} h_{s}^{-1} k\left(\left(z_{s}^{c}-X_{j s}^{c}\right) / h_{s}\right) d z_{s}^{c}$, where $k(\cdot)$ is a univariate density kernel function for a continuous variable such as the standard Epanechnikov or Gaussian kernel function. ${ }^{3}$ The cumulative kernel function for the vector of mixed variables is simply the product of $K_{h}(\cdot)$ and $L_{\lambda}(\cdot)$ defined above and is given by $G_{\gamma}\left(x, X_{j}\right)=K_{h}\left(x^{c}, X_{j}^{c}\right) \times L_{\lambda}\left(x^{d}, X_{j}^{d}\right)$, where $\gamma=(h, \lambda)$. Li et al. (2017) consider the mixeddatatype kernel estimator of $F_{X}(x)$ defined by

$$
\hat{F}_{X}(x)=\frac{1}{n} \sum_{j=1}^{n} G_{\gamma}\left(x, X_{j}\right) .
$$

Next, to estimate $F_{\epsilon}(t)$, we assume that $\epsilon$ is continuous and hence $F_{\epsilon}(t)$ can be estimated by a (univariate) continuous cumulative kernel estimator. However, unlike the case considered by Li et al. (2017), $\epsilon$ is not observed, so we first need to estimate it. To estimate $\mu(x)$ and $\sigma(x)$, we use local polynomial smoothing for the continuous covariates (see Fan \& Gijbels (1996) or Ruppert \& Wand (1994), among others), and for the discrete covariates, we use a variation on Aitchison \& Aitken (1976)'s kernel function defined by

$$
v\left(x_{s}^{d}, X_{j s}^{d}, \nu_{s}\right)= \begin{cases}1 & \text { if } x_{s}^{d}=X_{j s}^{d} \\ \nu_{s} & \text { otherwise. }\end{cases}
$$

The range of $\nu_{s}$ is $[0,1]$. Note that when $\nu_{s}=0$ the above kernel function becomes an indicator function, and when $\nu_{s}=1$, it is a constant function. The product kernel function for the vector $x^{d}$

\footnotetext{
${ }^{3}$ As noted by a referee, by working with kernel estimators we obtain a test that is not invariant to transformations of the regressors. A remedy could be to replace $k\left(\left(x_{s}^{c}-X_{s}^{c}\right) / h_{s}\right)$ by nearest neighbor windows of the form $k\left(\left(\tilde{F}_{X_{s}^{c}}\left(x_{s}^{c}\right)-\right.\right.$ $\left.\tilde{F}_{X_{s}^{c}}\left(X_{s}^{c}\right)\right) / h_{s}$ ), where $\tilde{F}_{X_{s}^{c}}$ is the non-smoothed empirical distribution of $X_{s}^{c}$. It is clear that this is invariant under any monotone transformation of $X_{s}^{c}$.
} 
of discrete covariates is then given by

$$
V_{\nu}\left(x^{d}, X_{j}^{d}\right)=\prod_{s=1}^{r} \nu_{s}^{1-1\left(x_{s}^{d}=X_{j s}^{d}\right)}
$$

Combining this with local polynomial smoothing of the continuous covariates (of which the order $p$ will depend on the dimension $q$ and will be determined later - see assumption (A2) in Appendix A), we define $\hat{\mu}(x)=\hat{\beta}_{0}$, where $\hat{\beta}_{0}$ is the first component of the vector $\hat{\beta}$, which is the solution of the local minimization problem

$$
\min _{\beta} \sum_{j=1}^{n}\left\{Y_{j}-P_{j}\left(\beta, x^{c}, p\right)\right\}^{2} \prod_{s=1}^{q} \frac{1}{g_{s}} k\left(\frac{x_{s}^{c}-X_{j s}^{c}}{g_{s}}\right) V_{\nu}\left(x^{d}, X_{j}^{d}\right)
$$

where $P_{j}\left(\beta, x^{c}, p\right)$ is a polynomial of order $p$ built up with all $0 \leq k \leq p$ products of factors of the form $X_{j s}^{c}-x_{s}^{c}(s=1, \ldots, q)$. The vector $\beta$ is the vector of length $\sum_{k=0}^{p} q^{k}$, consisting of all coefficients of this polynomial. Here, $g=\left(g_{1}, \ldots, g_{q}\right)$ is a $q$-dimensional bandwidth vector. To estimate $\sigma^{2}(x)$, define $\hat{\sigma}^{2}(x)=\hat{\gamma}_{0}$, where $\hat{\gamma}_{0}$ is defined in the same way as $\hat{\beta}_{0}$, but with $Y_{j}$ replaced by $\left(Y_{j}-\hat{\mu}\left(X_{j}\right)\right)^{2}$ in $(3)(j=1, \ldots, n)$.

Then, let $\hat{\epsilon}_{j}=\left(Y_{j}-\hat{\mu}\left(X_{j}\right)\right) / \hat{\sigma}\left(X_{j}\right)$ be the $j$-th residual, and define

$$
\hat{F}_{\hat{\epsilon}}(t)=\frac{1}{n} \sum_{j=1}^{n} \int_{-\infty}^{t} \frac{1}{b} k\left(\frac{s-\hat{\epsilon}_{j}}{b}\right) d s,
$$

where $b=b_{n}$ is the bandwidth for smoothing the residuals. Finally, let

$$
\hat{F}_{X, \hat{\epsilon}}(x, t)=\frac{1}{n} \sum_{j=1}^{n} G_{\gamma}\left(x, X_{j}\right) \int_{-\infty}^{t} \frac{1}{b} k\left(\frac{s-\hat{\epsilon}_{j}}{b}\right) d s
$$

be an estimator of the joint distribution $F_{X, \epsilon}(x, t)$ of $(X, \epsilon)$.

Bandwidth selection proceeds via minimization of a cross-validation function, which we explain for the distribution $F_{X}$ (for $F_{\epsilon}$ and $F_{X, \epsilon}$ similar ideas apply):

$$
C V(\gamma)=\frac{1}{n n_{j}} \sum_{i=1}^{n} \sum_{j=1}^{n_{j}}\left\{\mathbf{1}\left(X_{i} \leq x_{j}^{e}\right)-\hat{F}_{X,-i}\left(x_{j}^{e}\right)\right\}^{2},
$$

where $x_{j}^{e}, j=1, \ldots, n_{j}$, denotes evaluation points, and where $\hat{F}_{X,-i}(x)$ is the estimator defined in (2) except that the $i$-th data point is removed from the sample. The number of evaluation 
points can be fixed at, say, $n_{j}=100$. This grid of evaluation points plays a role not unlike the number/position of points used for numerical integration. Under quite general conditions and using the cross-validated bandwidths, Li et al. (2017) obtain the result that

$$
\sqrt{n}\left(\hat{F}_{X}(x)-F_{X}(x)-\frac{\kappa_{2}}{2} \sum_{s=1}^{q} h_{s}^{2} \frac{\partial^{2}}{\partial\left(x_{s}^{c}\right)^{2}} F_{X}(x)-\sum_{s=1}^{r} \lambda_{s} B_{s}(x)\right) \stackrel{d}{\rightarrow} N(0, V),
$$

where $V=F(x)(1-F(x)), \kappa_{2}=\int u^{2} k(u) d u$, and

$$
B_{s}(x)=E_{X^{d}}\left[\sum_{z^{d} \leq x^{d}} \mathbf{1}\left(X_{-s}^{d}=z_{-s}^{d}\right) P\left(X_{s}^{d}=z_{s}^{d}\right) F_{X^{c} \mid X^{d}}\left(x^{c} \mid X^{d}\right)\right],
$$

with $F_{X^{c} \mid X^{d}}\left(x^{c} \mid x^{d}\right)$ the conditional distribution of $X^{c}$ given $X^{d}, X_{-s}^{d}$ contains all components of $X^{d}$ except the $s$-th component, and equalities and inequalities should be understood componentwise.

Li et al. (2017) deliver a smooth nonparametric estimator that, like its non-smooth EDF counterpart, achieves a dimension-free $\sqrt{n}$ rate of convergence. The important point to note is that when the underlying distribution is itself smooth, the kernel estimator is capable of delivering estimators that outperform their non-smooth counterparts in finite-sample settings; see Li et al. (2017) for details. In a typical location-scale model with a continuous response and predictor, smoothness of the joint and marginal distributions of the predictor and error term can be safely assumed in a wide range of applications.

\section{Asymptotic Properties}

We start with a preliminary result that gives an iid representation for the estimators $\hat{F}_{X}(x)$, $\hat{F}_{\hat{\epsilon}}(t)$ and $\hat{F}_{X, \hat{\epsilon}}(x, t)$. The regularity conditions mentioned below, as well as the proofs of the results of this section, can be found in Appendix A.

Define $\kappa_{2}=\int u^{2} k(u) d u$, and more generally for $p \geq 0$, let $\kappa_{p+1}$ be the first element of the vector $S^{-1}\left(s_{p+1}, \ldots, s_{2 p+1}\right)^{T}$, where $S$ is the $(p+1) \times(p+1)$ matrix whose $(i, j)$-th entry is $s_{i+j-2}$, with $s_{j}=\int u^{j} k(u) d u$. Also, let

$$
C_{s}(x)=\sum_{z^{d}}\left[1\left(z_{s}^{d} \neq x_{s}^{d}\right) \prod_{t \neq s} 1\left(z_{t}^{d}=x_{t}^{d}\right) \mu\left(x^{c}, z^{d}\right)-\mu(x)\right] f_{X}\left(x^{c}, z^{d}\right)
$$




$$
D_{s}(x)=\sum_{z^{d}}\left[1\left(z_{s}^{d} \neq x_{s}^{d}\right) \prod_{t \neq s} 1\left(z_{t}^{d}=x_{t}^{d}\right) \sigma^{2}\left(x^{c}, z^{d}\right)-\sigma^{2}(x)\right] f_{X}\left(x^{c}, z^{d}\right),
$$

for $s=1, \ldots, r$, where $f_{X}(x)$ is the joint probability density function of $X$.

Theorem 1. Assume (A1)-(A6). Then, under $H_{0}$ and for any $x$ and $t$,

$$
\begin{aligned}
\hat{F}_{X}(x)-F_{X}(x) & =\frac{1}{n} \sum_{i=1}^{n} \mathbf{1}\left(X_{i} \leq x\right)-F_{X}(x)+\frac{\kappa_{2}}{2} \sum_{s=1}^{q} h_{s}^{2} \frac{\partial^{2} F_{X}(x)}{\partial\left(x_{s}^{c}\right)^{2}}+\sum_{s=1}^{r} \lambda_{s} B_{s}(x)+R_{n, X}(x), \\
\hat{F}_{\hat{\epsilon}}(t)-F_{\epsilon}(t) & =\frac{1}{n} \sum_{i=1}^{n}\left[1\left(\epsilon_{i} \leq t\right)-F_{\epsilon}(t)+f_{\epsilon}(t)\left\{\epsilon_{i}+\frac{t}{2}\left(\epsilon_{i}^{2}-1\right)\right\}\right] \\
& +f_{\epsilon}(t) \int \frac{1}{\sigma(z)}\left\{\frac{\kappa_{p+1}}{(p+1) !} \sum_{s=1}^{q} g_{s}^{p+1} \frac{\partial^{p+1}}{\partial\left(z_{s}^{c}\right)^{p+1}} \mu(z)+\sum_{s=1}^{r} \nu_{s} C_{s}(z)\right\} d F_{X}(z) \\
& +\frac{t}{2} f_{\epsilon}(t) \int \frac{1}{\sigma^{2}(z)}\left\{\frac{\kappa_{p+1}}{(p+1) !} \sum_{s=1}^{q} g_{s}^{p+1} \frac{\partial^{p+1}}{\partial\left(z_{s}^{c}\right)^{p+1}} \sigma^{2}(z)+\sum_{s=1}^{r} \nu_{s} D_{s}(z)\right\} d F_{X}(z) \\
& +\frac{\kappa_{2}}{2} b^{2} f_{\epsilon}^{\prime}(t)+R_{n, \epsilon}(t), \\
\hat{F}_{X, \hat{\epsilon}}(x, t)-F_{X, \epsilon}(x, t) & =\frac{1}{n} \sum_{i=1}^{n}\left[\mathbf{1}\left(X_{i} \leq x, \epsilon_{i} \leq t\right)-F_{X, \epsilon}(x, t)+f_{\epsilon}(t) \mathbf{1}\left(X_{i} \leq x\right)\left\{\epsilon_{i}+\frac{t}{2}\left(\epsilon_{i}^{2}-1\right)\right\}\right] \\
& +f_{\epsilon}(t) \int_{-\infty}^{x} \frac{1}{\sigma(z)}\left\{\frac{\kappa_{p+1}}{(p+1) !} \sum_{s=1}^{q} g_{s}^{p+1} \frac{\partial^{p+1}}{\partial\left(z_{s}^{c}\right)^{p+1}} \mu(z)+\sum_{s=1}^{r} \nu_{s} C_{s}(z)\right\} d F_{X}(z) \\
& +\frac{t}{2} f_{\epsilon}(t) \int_{-\infty}^{x} \frac{1}{\sigma^{2}(z)}\left\{\frac{\kappa_{p+1}}{(p+1) !} \sum_{s=1}^{q} g_{s}^{p+1} \frac{\partial^{p+1}}{\partial\left(z_{s}^{c}\right)^{p+1}} \sigma^{2}(z)+\sum_{s=1}^{r} \nu_{s} D_{s}(z)\right\} d F_{X}(z) \\
& +\frac{\kappa_{2}}{2} \sum_{s=1}^{q} h_{s}^{2} \frac{\partial^{2} F_{X}(x)}{\partial\left(x_{s}^{c}\right)^{2}} F_{\epsilon}(t)+\sum_{s=1}^{r} \lambda_{s} B_{s}(x) F_{\epsilon}(t)+\frac{\kappa_{2}}{2} b^{2} F_{X}(x) f_{\epsilon}^{\prime}(t) \\
& +R_{n, X, \epsilon}(x, t),
\end{aligned}
$$

where $\sup _{x \in R_{X}}\left|R_{n, X}(x)\right|=o_{P}\left(n^{-1 / 2}\right), \sup _{t \in \mathcal{R}}\left|R_{n, \epsilon}(t)\right|=o_{P}\left(n^{-1 / 2}\right)$ and $\sup _{x \in R_{X}, t \in \mathcal{R}}\left|R_{n, X, \epsilon}(x, t)\right|=$ $o_{P}\left(n^{-1 / 2}\right)$.

An immediate consequence of this theorem is the following corollary. Note that instead of assuming condition (A3) which says that $h, \lambda$ and $b$ should tend to zero sufficiently fast, we only require that $h, \lambda$, and $b \rightarrow 0$. This is because the bias coming from the smoothing of the empirical distribution functions disappears in the formula of $\hat{F}_{X, \hat{\epsilon}}(x, t)-\hat{F}_{X}(x) \hat{F}_{\hat{\epsilon}}(t)$. 
Corollary 2. Assume $h, \lambda, b \rightarrow 0,(A 1)-(A 2)$ and (A4)-(A6). Then, under $H_{0}$ and for any $x$ and $t$,

$$
\sqrt{n}\left(\hat{F}_{X, \hat{\epsilon}}(x, t)-\hat{F}_{X}(x) \hat{F}_{\hat{\epsilon}}(t)\right)=\frac{1}{\sqrt{n}} \sum_{i=1}^{n} H\left(X_{i}, \epsilon_{i}, x, t\right)+b(x, t)+R_{n}(x, t)
$$

where

$$
\begin{gathered}
H\left(X_{i}, \epsilon_{i}, x, t\right)=\mathbf{1}\left(X_{i} \leq x, \epsilon_{i} \leq t\right)-F_{X, \epsilon}(x, t)-F_{\epsilon}(t)\left\{\mathbf{1}\left(X_{i} \leq x\right)-F_{X}(x)\right\} \\
-F_{X}(x)\left\{1\left(\epsilon_{i} \leq t\right)-F_{\epsilon}(t)\right\}+f_{\epsilon}(t)\left\{\mathbf{1}\left(X_{i} \leq x\right)-F_{X}(x)\right\}\left\{\epsilon_{i}+\frac{t}{2}\left(\epsilon_{i}^{2}-1\right)\right\}, \\
b(x, t)=f_{\epsilon}(t) \int\left(\mathbf{1}(z \leq x)-F_{X}(x)\right) \frac{1}{\sigma(z)}\left\{\frac{\kappa_{p+1}}{(p+1) !} \sum_{s=1}^{q} c_{g, s}^{p+1} \frac{\partial^{p+1}}{\partial\left(z_{s}^{c}\right)^{p+1}} \mu(z)+\sum_{s=1}^{r} c_{\nu, s} C_{s}(z)\right\} d F_{X}(z) \\
+\frac{t}{2} f_{\epsilon}(t) \int\left(\mathbf{1}(z \leq x)-F_{X}(x)\right) \frac{1}{\sigma^{2}(z)}\left\{\frac{\kappa_{p+1}}{(p+1) !} \sum_{s=1}^{q} c_{g, s}^{p+1} \frac{\partial^{p+1}}{\partial\left(z_{s}^{c}\right)^{p+1}} \sigma^{2}(z)+\sum_{s=1}^{r} c_{\nu, s} D_{s}(z)\right\} d F_{X}(z),
\end{gathered}
$$

and $\sup _{x \in R_{X}, t \in \mathcal{R}}\left|R_{n}(x, t)\right|=o_{P}(1)$.

Remark 3. Note that some of the bias terms appearing in Theorem 1 cancel out in Corollary 2. Indeed, the biases arising from smoothing the distribution functions $\hat{F}_{X}(x), \hat{F}_{\hat{\epsilon}}(t)$ and $\hat{F}_{X, \hat{\epsilon}}(x, t)$, disappeared in Corollary 2. Hence, the asymptotic representation of $\sqrt{n}\left(\hat{F}_{X, \hat{\epsilon}}(x, t)-\hat{F}_{X}(x) \hat{F}_{\hat{\epsilon}}(t)\right)$ is the same as in the case where the empirical distributions are not smoothed (see Einmahl $\&$ Van Keilegom (2008)). However, inspection of the proof of Theorem 2.2 in the latter paper reveals that their iid expansion of $\sqrt{n}\left(\hat{F}_{X, \hat{\epsilon}}(x, t)-\hat{F}_{X}(x) \hat{F}_{\hat{\epsilon}}(t)\right)$ does not contain the term $f_{\epsilon}(t) n^{-1 / 2} \sum_{i=1}^{n}\left\{\mathbf{1}\left(X_{i} \leq\right.\right.$ $\left.x)-F_{X}(x)\right\}\left\{\epsilon_{i}+\frac{t}{2}\left(\epsilon_{i}^{2}-1\right)\right\}$ that shows up in the formula of $n^{-1 / 2} \sum_{i=1}^{n} H\left(X_{i}, \epsilon_{i}, x, t\right)$ given above. This is because the second statement in their Lemma A.1 is wrong, in the sense that the expression on the left hand side is not centered, and so it is certainly not $o_{P}\left(n^{-1 / 2}\right)$. The correct version of their Theorem 2.2 can be obtained from Corollary 4 below by using undersmoothing of the bandwidth $g_{1}$ (and taking $q=1$ and $r=0$ ).

We are now ready to state the weak convergence of $\sqrt{n}\left(\hat{F}_{X, \hat{\epsilon}}-\hat{F}_{X} \hat{F}_{\hat{\epsilon}}\right)$ as a process in $\ell^{\infty}\left(R_{X} \times \mathcal{R}\right)$, and the limiting distribution of our test statistics $T_{K S}$ and $T_{C M}$. Here, $\ell^{\infty}\left(R_{X} \times \mathcal{R}\right)$ is the set of bounded functions from $R_{X} \times \mathcal{R}$ to $\mathcal{R}$, equipped with the uniform norm.

Corollary 4. Assume $h, \lambda, b \rightarrow 0$, (A1)-(A2) and (A4)-(A6). 
(i) Under $H_{0}$, the process $\sqrt{n}\left(\hat{F}_{X, \hat{\epsilon}}(x, t)-\hat{F}_{X}(x) \hat{F}_{\hat{\epsilon}}(t)\right)$ converges weakly in $\ell^{\infty}\left(R_{X} \times \mathcal{R}\right)$ to a Gaussian process $Z(x, t)$ with mean function $b(x, t)$ and covariance function

$$
\operatorname{Cov}\left(Z\left(x_{1}, t_{1}\right), Z\left(x_{2}, t_{2}\right)\right)=E\left[H\left(X, \epsilon, x_{1}, t_{1}\right) H\left(X, \epsilon, x_{2}, t_{2}\right)\right]
$$

for $x_{1}, x_{2} \in R_{X}$ and $t_{1}, t_{2} \in \mathcal{R}$.

(ii) Under $H_{0}$,

$$
T_{K S} \stackrel{d}{\rightarrow} \sup _{x \in R_{X}, t \in \mathcal{R}}|Z(x, t)| \quad \text { and } \quad T_{C M} \stackrel{d}{\rightarrow} \iint Z^{2}(x, t) d F_{X}(x) d F_{\epsilon}(t)
$$

\section{Second Order Properties}

We now analyze the source of the power gains that arise from smoothing by considering higher order expansions (we are grateful to an anonymous referee who suggested that we address this issue). Below we demonstrate that the smoothed process has smaller MISE than the non-smoothed process which arises primarily due to a reduction in variance, which in turn leads to the finitesample power gains that are evident in the simulations reported in Section 5 below. For notational convenience we shall restrict attention to the case of one continuous covariate (so $q=1$ and $r=0$ ), but the result can be readily extended to the general case.

We use the notations $\tilde{F}_{\hat{\epsilon}}(t)=n^{-1} \sum_{i=1}^{n} 1\left(\hat{\epsilon}_{i} \leq t\right)$ and $\tilde{F}_{X, \hat{\epsilon}}(x, t)=n^{-1} \sum_{i=1}^{n} 1\left(X_{i} \leq x, \hat{\epsilon}_{i} \leq t\right)$ for the non-smoothed estimators of $F_{\epsilon}(t)$ and $F_{X, \epsilon}(x, t)$ respectively.

Theorem 5 below demonstrates that the MISE of the smoothed process dominates that of the non-smoothed process, and the reduction in MISE translates into improved power for our proposed procedure.

Theorem 5. Assume $h, b \rightarrow 0, n g^{7}(\log n)^{-1} \rightarrow \infty, p \geq 3$, (A1)-(A2) and (A4)-(A6). Then,

$$
\begin{aligned}
n^{1 / 2}\left(\hat{F}_{X, \hat{\epsilon}}(x, t)-\hat{F}_{X}(x) \hat{F}_{\hat{\epsilon}}(t)\right) & =n^{-1 / 2} \sum_{i=1}^{n} \iint H\left(X_{i}, \epsilon_{i}, x-u h, t-v b\right) k(u) k(v) d u d v+b(x, t) \\
& +\hat{R}(x, t) \\
& :=\hat{G}(x, t)+b(x, t)+\hat{R}(x, t) \\
n^{1 / 2}\left(\tilde{F}_{X, \hat{\epsilon}}(x, t)-\tilde{F}_{X}(x) \tilde{F}_{\hat{\epsilon}}(t)\right) & =n^{-1 / 2} \sum_{i=1}^{n} H\left(X_{i}, \epsilon_{i}, x, t\right)+b(x, t)+\tilde{R}(x, t)
\end{aligned}
$$




$$
:=\tilde{G}(x, t)+b(x, t)+\tilde{R}(x, t),
$$

where $\sup _{x, t}|\hat{R}(x, t)|=O_{P}\left((n g)^{-1 / 6} \log n\right)$ and $\sup _{x, t}|\tilde{R}(x, t)|=O_{P}\left((n g)^{-1 / 6} \log n\right)$.

In addition,

$$
\begin{aligned}
& \int \operatorname{Var}(\hat{G}(x, t)) d x d t=[A-h \Psi(k)][B-b \Psi(k)]+O\left(h^{2}+b^{2}\right) \\
& \int \operatorname{Var}(\tilde{G}(x, t)) d x d t=A B+O\left(h^{2}+b^{2}\right),
\end{aligned}
$$

where

$$
\begin{aligned}
A & =\int F_{X}(x)\left(1-F_{X}(x)\right) d x>0 \\
B & =\int\left\{F_{\epsilon}(t)\left(1-F_{\epsilon}(t)\right)+E\left[2\left\{I(\epsilon \leq t)-F_{\epsilon}(t)\right\} Q(t, \epsilon)+Q^{2}(t, \epsilon)\right]\right\} d t>0 \\
Q(t, \epsilon) & =f_{\epsilon}(t)\left\{\epsilon+\frac{t}{2}\left(\epsilon^{2}-1\right)\right\} \\
\Psi(k) & =2 \int u K(u) k(u) d u>0 .
\end{aligned}
$$

Note that the remainder terms $\hat{R}(x, t)$ and $\tilde{R}(x, t)$ are of the order $O_{P}\left(n^{-1 / 6} n^{1 /[6(2 p+2)]} \log n\right)$ uniformly in $x$ and $t$ if we take $c_{g, s}>0$ in condition (A2). Since $p \geq 3$, this is $O_{P}\left(n^{-7 / 48} \log n\right)$ in the worst case (i.e., when $p=3$ ). On the other hand, if $h$ is e.g. proportional to $n^{-1 / 4}$ (but any larger bandwidth is also fine), then $h^{1 / 2} \sim n^{-1 / 8}$, and this is larger than $n^{-7 / 48} \log n$ (and similarly for $\left.b^{1 / 2}\right)$. Hence, the remainder terms are of negligible order. Since $\Psi(k), A, B>0$ the above result shows that the smoothed process has smaller MISE than the non-smoothed process. The proof of Theorem 5 can be found in Appendix A.

The astute reader may have noted that the first term in the expansion of $\sqrt{n}\left(\hat{F}_{X, \hat{\epsilon}}-\hat{F}_{X} \hat{F}_{\hat{\epsilon}}\right)$ differs from that in Corollary 2, and perhaps a few words are in order. In Corollary 2 we were only interested in first-order asymptotic properties, and so we only needed to demonstrate that the order of the remainder term is $o_{P}(1)$. In Theorem 5 however, we want to show that the smoothed estimator has better second-order properties than the non-smoothed estimator, and that requires a finer analysis. The main term in the i.i.d. expansion in Theorem 5 is actually equal to the main term in the i.i.d. expansion in Corollary 2, except for some terms that are of smaller order and that are absorbed in the remainder term $R_{n}$ in Corollary 2. In Theorem 5 we cannot absorb these 
terms in the remainder term simply because we need them to show that the smoothed estimator outperforms the non-smoothed one in second order. This explains why the two representations are different.

\section{Finite-Sample Performance}

5.1. The Univariate Continuous Predictor Setting. In order to assess the finite-sample performance of our proposed approach, we replace the EDFs in (1) with their kernel-smoothed counterparts described in Section 2.1. ${ }^{4}$ Bandwidth selection is obtained via cross-validation for $h, b$ and $\lambda$ (i.e., minimization of Equation (6) above) and via least squares cross-validation for $g$ and $\nu$, and the Epanechnikov kernel function is employed. There are various data-driven permutations one might consider for bandwidth selection, namely a) separate bandwidths for the joint and marginal distributions, b) common bandwidths taken from the joint distribution, and c) common bandwidths taken from the marginal distributions. From the perspective of assessing size, one might expect that the same bandwidths used for estimating the joint distribution be used for the construction of the marginal distributions, otherwise the estimated joint distribution might systematically diverge from the product of the estimated marginals under the null. We investigate this issue empirically and on this basis recommend b) to practitioners.

To assess finite-sample performance, we simulate data for which $X$ is uniform [0,1] and $Y$ has location $\mu(x)=\sin (2 \pi x)$ and scale $\sigma(x)$ that is determined from the error distributions specified below, i.e.,

$$
Y_{i}=\sin \left(2 \pi X_{i}\right)+\sigma\left(X_{i}\right) \epsilon_{i}\left(X_{i}\right)
$$

We consider three DGPs in the simulations that follow. For the first the errors are mixtures of two Gaussians, $N\left(-1,0.5^{2}\right)$ and $N\left(1,0.5^{2}\right)$ with mixing probabilities $1-\delta x$ and $\delta x$. For the second the errors are drawn from a heavy-tail mixture of two $t$-distributions, one having a mode at 2 and the other at -2 , both having 5 degrees of freedom with the same mixing probabilities as for the Gaussian mixture. For the third the errors are drawn from the Beta distribution with shape parameters $s_{1}=1+\delta 10 x$ and $s_{2}=11-\delta 10 x$ where $x \in[0,1]$. These errors are then rescaled

\footnotetext{
${ }^{4}$ We proceed with the Kolmogorov-Smirnov statistic by way of illustration as the Cramér-von-Mises statistic requires multivariate integration for its computation while the Kolmogorov-Smirnov statistic does not. However, as will be demonstrated for the Kolmogorov-Smirnov approach, replacing the non-smooth distribution functions in the Cramérvon-Mises statistic with their kernel-smoothed counterparts would be expected to reveal similar power gains.
} 
to have unconditional mean zero and unit variance thereby maintaining a constant signal-to-noise ratio across DGPs and across the range of values for $\delta$ considered. In all cases, when $\delta=0$ the model is a location-scale DGP (i.e., the distribution of $\epsilon$ is not a function of $x$ ) while when $\delta>0$ it is a location-shape DGP (i.e., the distribution of $\epsilon$ is a function of $x$ ). Figure 1 presents the density of $\epsilon(x)$ for various levels of $x \in[0,1]$ when $\delta=1$.

For what follows we consider $M=1,000$ Monte Carlo replications drawn from each DGP. For each Monte Carlo replication, we compute each test statistic $T_{K S}$ (non-smooth and smooth, respectively) along with the $B=399$ (Davidson \& MacKinnon 2000) null bootstrap replicates $T_{b, K S}^{*}, b=1, \ldots, B$ (based on resamples drawn from the (non-smooth) distribution functions), then we compute the empirical $P$-value as $B^{-1} \sum_{b=1}^{B} \mathbf{1}\left(T_{b, K S}^{*}>T_{K S}\right)$, where $\mathbf{1}(A)$ is the usual indicator function taking value one when $A$ is true and zero otherwise. Finally, based on the $M=1,000 P$-values, we compute empirical rejection probabilities for the non-smooth and smooth test statistics for nominal levels $\alpha=(0.01,0.05,0.10)$. To assess size we set $\delta=0$, and to assess power we let $\delta \in(0,1]$. Size (i.e., empirical rejection probability when $\delta=0)$ is summarized in Table 1.

Table 1 indicates that both the non-smooth and smooth versions of the test appear to be correctly sized, hence we can proceed to compare their power curves. ${ }^{5}$ Next, we vary $\delta \in[0,1]$, and present power curves for the Beta errors in Figure 4 (Figures 2 and 3 present results for the Gaussian and Student- $t$ mixtures).

The percentage gain in power is reported in the tables in Appendix B, and these figures and tables reveal that the improvements in power arising from replacing the EDF with its kernel-smoothed counterpart can be upwards of $100 \%$ or more, depending on the nominal size of the test, sample size, and degree of departure from the null. Furthermore, if anything the smooth test appears to be slightly conservative relative to its non-smooth counterpart, particularly for the Beta error case for the smaller sample sizes considered (a positive feature as it has a lower probability of a Type I error than its non-smooth counterpart under the null yet higher power under the alternative).

\footnotetext{
${ }^{5}$ If anything, the non-smooth version appears to be slightly over-sized for smaller $n$ and the smooth version slightly under-sized for smaller $n$, but this admits a fair comparison of power curves.
} 

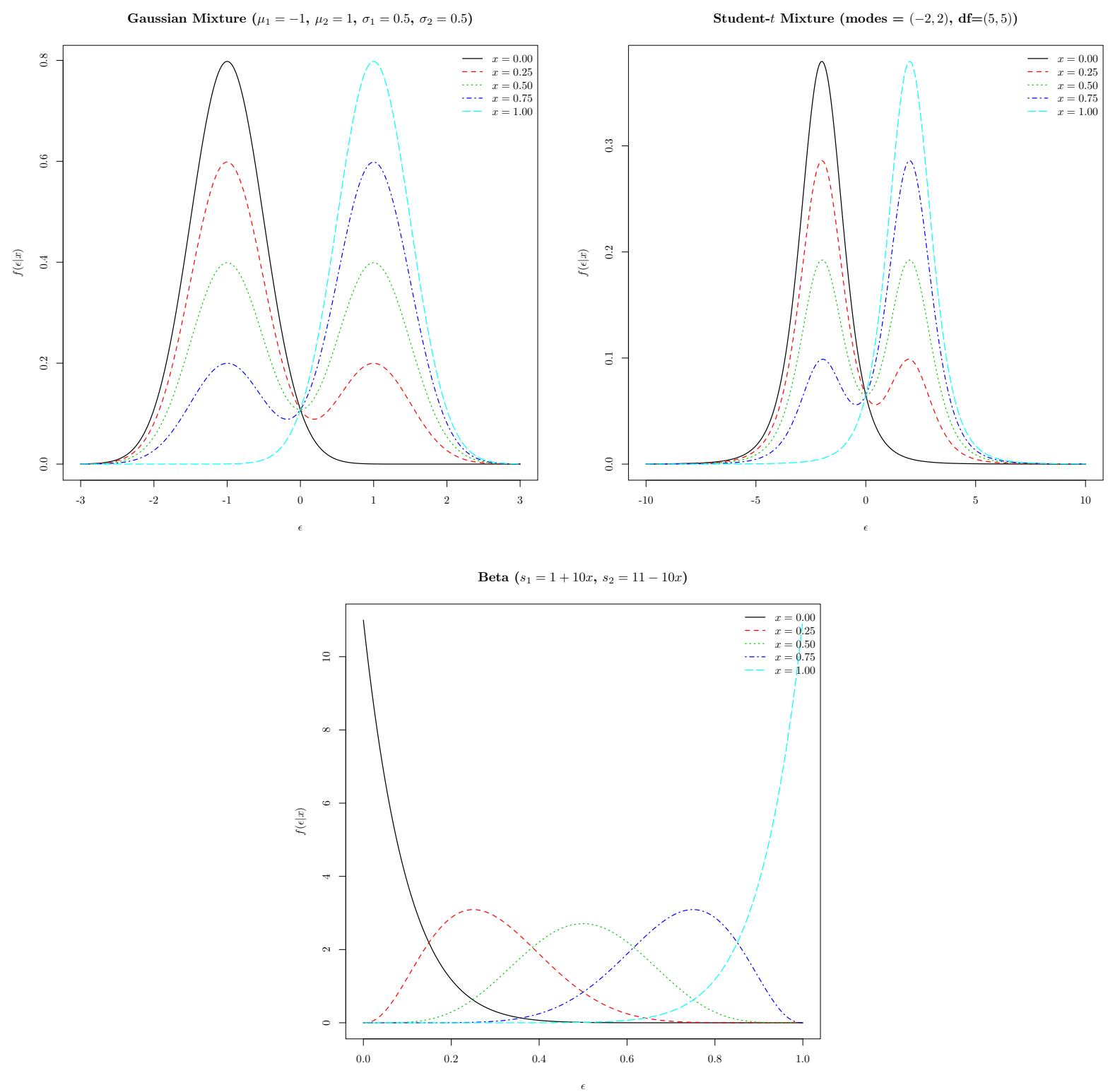

Figure 1. Density of $\epsilon(x)$ for various levels of $x \in[0,1], \delta=1$, for the Gaussian mixture, Student- $t$ mixture and Beta (the error density for computing size, $\delta=0$, corresponds to the density when $x=0$ i.e., the solid black density).

5.2. The Multivariate Continuous Predictor Setting. To assess finite-sample performance in the multivariate continuous predictor setting, we simulate data for which $X_{1}$ and $X_{2}$ are uniform $[0,1]$ and $Y$ has location $\mu(x)=\sin \left(\pi\left(x_{1}+x_{2}\right)\right)$ and scale $\sigma\left(x_{1}+x_{2}\right) / 2$ that is determined from 
TABle 1. Size for the Non-smooth and Smooth Tests (Empirical Rejection Probabilities Under the Null, $\delta=0$, Univariate Continuous Predictor Setting, see Appendix B for Power).

\begin{tabular}{|c|c|c|c|}
\hline$n$ & $\alpha=0.01$ & $\alpha=0.0$ & $\alpha=0.10$ \\
\hline \multicolumn{4}{|c|}{ Non-smooth, Gaussian Mixture } \\
\hline 100 & 0.008 & 0.041 & 0.101 \\
\hline 200 & 0.008 & 0.045 & 0.089 \\
\hline 400 & 0.006 & 0.048 & 0.089 \\
\hline 800 & 0.006 & 0.045 & 0.102 \\
\hline \multicolumn{4}{|c|}{ Smooth, Gaussian Mixture } \\
\hline 100 & 0.011 & 0.045 & 0.104 \\
\hline 200 & 0.006 & 0.046 & 0.093 \\
\hline 400 & 0.007 & 0.057 & 0.110 \\
\hline 800 & 0.010 & 0.047 & 0.092 \\
\hline \multicolumn{4}{|c|}{ Non-smooth, Student- $t$ Mixture } \\
\hline 100 & 0.009 & 0.051 & 0.081 \\
\hline 200 & 0.009 & 0.050 & 0.104 \\
\hline 400 & 0.005 & 0.039 & 0.095 \\
\hline 800 & 0.006 & 0.038 & 0.089 \\
\hline \multicolumn{4}{|c|}{ Smooth, Student- $t$ Mixture } \\
\hline 100 & 0.009 & 0.048 & 0.101 \\
\hline 200 & 0.010 & 0.052 & 0.095 \\
\hline 400 & 0.010 & 0.045 & 0.092 \\
\hline 800 & 0.008 & 0.042 & 0.095 \\
\hline \multicolumn{4}{|c|}{ Non-smooth, Beta } \\
\hline 100 & 0.012 & 0.049 & 0.090 \\
\hline 200 & 0.008 & 0.050 & 0.105 \\
\hline 400 & 0.008 & 0.038 & 0.099 \\
\hline 800 & 0.011 & 0.048 & 0.098 \\
\hline \multicolumn{4}{|c|}{ Smooth, Beta } \\
\hline 100 & 0.011 & 0.042 & 0.074 \\
\hline 200 & 0.012 & 0.051 & 0.095 \\
\hline 400 & 0.008 & 0.054 & 0.110 \\
\hline 800 & 0.013 & 0.054 & 0.104 \\
\hline
\end{tabular}

the error distributions specified below, i.e.,

$$
Y_{i}=\sin \left(\pi\left(X_{i 1}+X_{i 2}\right)\right)+\sigma\left(X_{i 1}+X_{i 2}\right) \epsilon_{i}\left(X_{i 1}+X_{i 2}\right)
$$

We consider three DGPs in the simulations that follow. For the first the errors are mixtures of two Gaussians, $N\left(-1,0.5^{2}\right)$ and $N\left(1,0.5^{2}\right)$ with mixing probabilities $1-\delta\left(x_{1}+x_{2}\right) / 2$ and $\delta\left(x_{1}+x_{2}\right) / 2$. For the second the errors are drawn from a heavy-tail mixture of two $t$-distributions, one having a mode at 2 and the other at -2 , both having 5 degrees of freedom, with the same mixing probabilities 

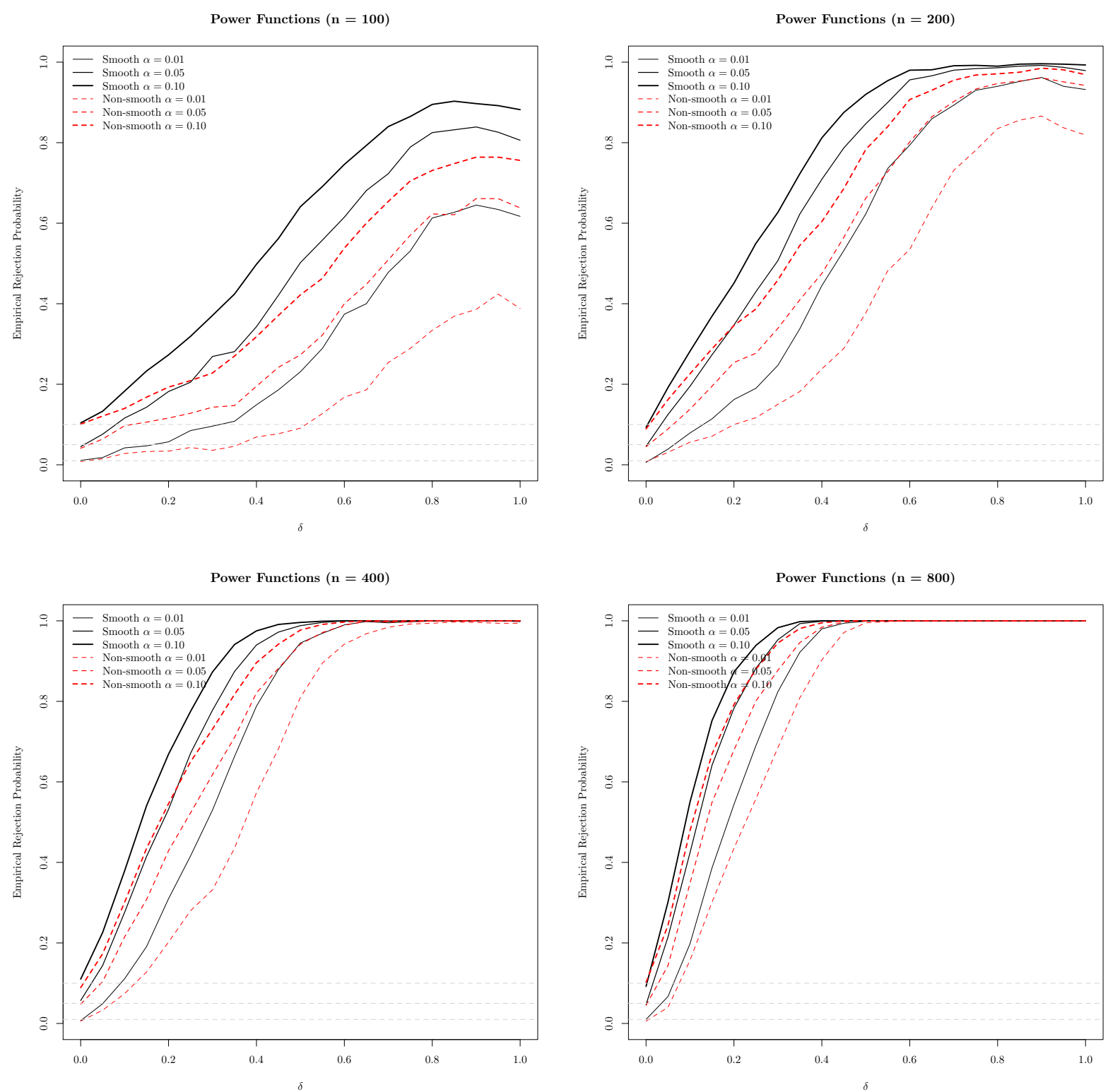

FiguRE 2. Power curves for the non-smooth and smooth versions of the test, Gaussian mixture. Solid lines are for the smooth version, dashed the non-smooth version.

as for the Gaussian mixture. For the third the errors are drawn from the Beta distribution with shape parameters $s_{1}=1+\delta 5\left(x_{1}+x_{2}\right)$ and $s_{2}=11-\delta 5\left(x_{1}+x_{2}\right)$. Per above, these errors are then rescaled to have unconditional mean zero and unit variance.

Table 2 reveals that both the non-smooth and smooth version of the test perform adequately and appear to possess reasonable size when there exist multivariate continuous predictors. 

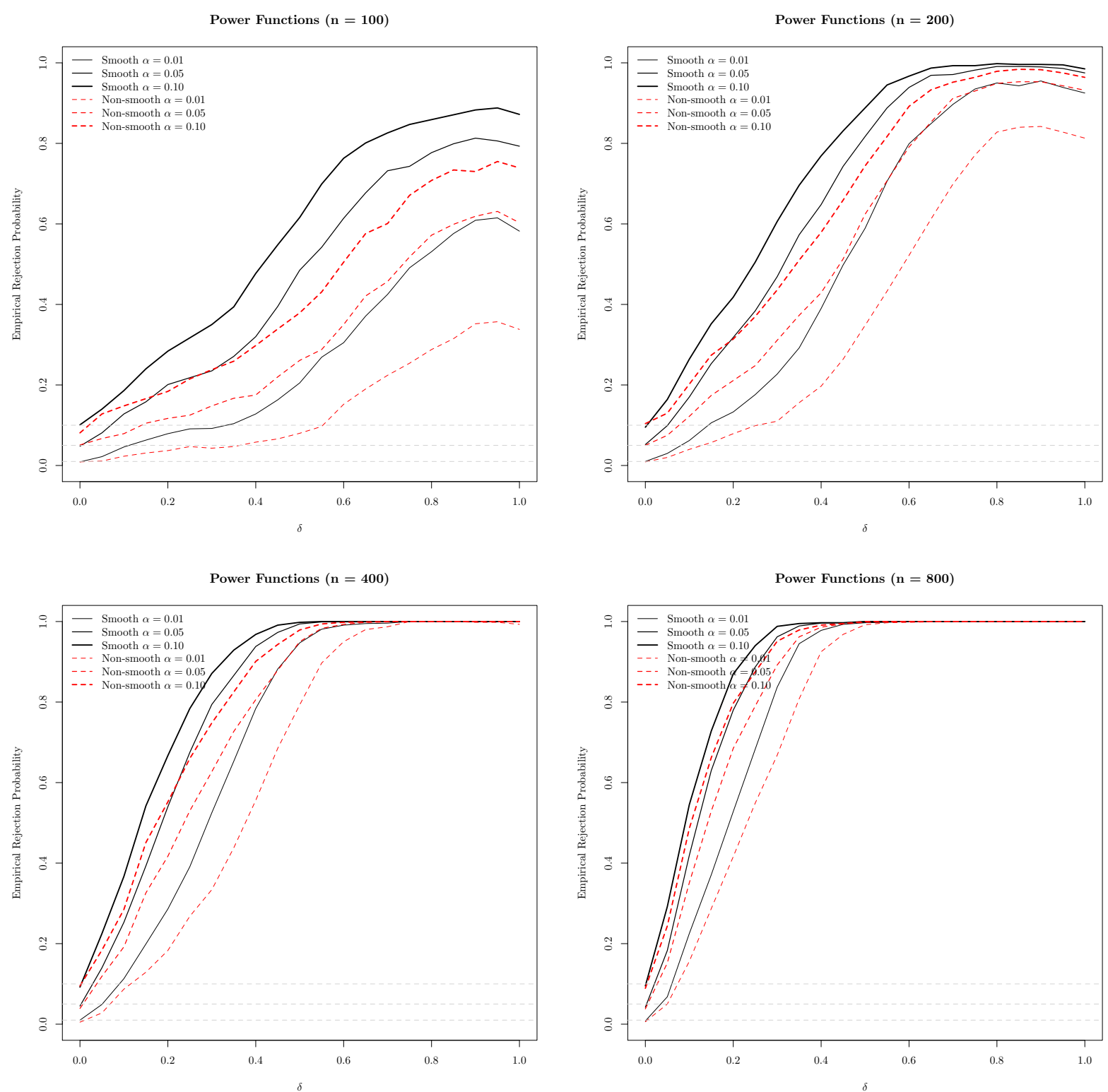

FiguRe 3. Power curves for the non-smooth and smooth versions of the test, Student- $t$ mixture. Solid lines are for the smooth version, dashed the non-smooth version.

\subsection{The Multivariate Mixed Continuous and Discrete Predictor Setting. To assess finite-} sample performance in the multivariate mixed continuous and discrete predictor setting, we simulate data for which $X_{1}$ is uniform $[0,1], X_{2}$ is a discrete uniform variable taking value $0,1 / 10,2 / 10, \ldots, 1$, $Y$ has location $\mu(x)=\sin \left(2 \pi x_{1}\right)+x_{2}$ and scale $\sigma\left(X_{i}\right)$ that is determined from the error distributions 

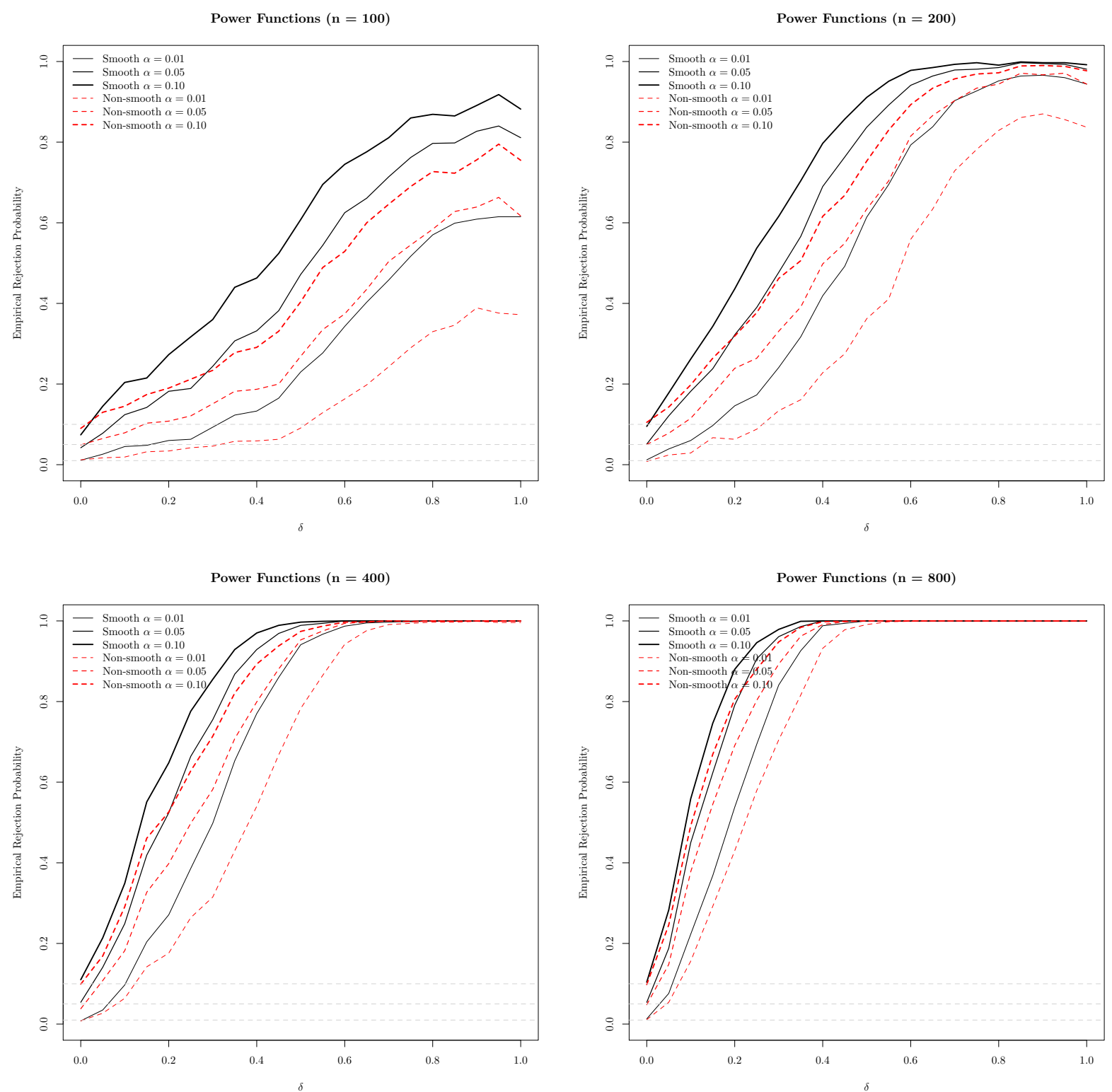

Figure 4. Power curves for the non-smooth and smooth versions of the test, Beta errors. Solid lines are for the smooth version, dashed the non-smooth version.

specified below, i.e.,

$$
Y_{i}=\sin \left(2 \pi X_{i 1}\right)+X_{i 2}+\sigma\left(X_{i 1}+X_{i 2}\right) \epsilon_{i}\left(X_{i 1}+X_{i 2}\right)
$$

We consider three DGPs in the simulations that follow. For the first the errors are mixtures of two Gaussians, $N\left(-1,0.5^{2}\right)$ and $N\left(1,0.5^{2}\right)$ with mixing probabilities $1-\delta\left(x_{1}+x_{2}\right) / 2$ and $\delta\left(x_{1}+x_{2}\right) / 2$. 
TABle 2. Size for the Non-smooth and Smooth Tests (Empirical Rejection Probabilities Under the Null, $\delta=0$, Multivariate Continuous Predictor Setting, see Appendix C for Power).

\begin{tabular}{|c|c|c|c|}
\hline$n$ & $\alpha=0.01$ & $\alpha=0.05$ & $\alpha=0.10$ \\
\hline \multicolumn{4}{|c|}{ Non-smooth, Gaussian Mixture } \\
\hline 100 & 0.007 & 0.042 & 0.100 \\
\hline 200 & 0.007 & 0.042 & 0.094 \\
\hline 400 & 0.012 & 0.038 & 0.075 \\
\hline 800 & 0.005 & 0.037 & 0.075 \\
\hline \multicolumn{4}{|c|}{ Smooth, Gaussian Mixture } \\
\hline 100 & 0.005 & 0.033 & 0.091 \\
\hline 200 & 0.008 & 0.048 & 0.087 \\
\hline 400 & 0.006 & 0.032 & 0.072 \\
\hline 800 & 0.006 & 0.037 & 0.073 \\
\hline \multicolumn{4}{|c|}{ Non-smooth, Student- $t$ Mixture } \\
\hline 100 & 0.018 & 0.056 & 0.116 \\
\hline 200 & 0.018 & 0.080 & 0.121 \\
\hline 400 & 0.012 & 0.053 & 0.107 \\
\hline 800 & 0.008 & 0.055 & 0.095 \\
\hline \multicolumn{4}{|c|}{ Smooth, Student- $t$ Mixture } \\
\hline 100 & 0.023 & 0.078 & 0.140 \\
\hline 200 & 0.016 & 0.085 & 0.140 \\
\hline 400 & 0.017 & 0.068 & 0.111 \\
\hline 800 & 0.012 & 0.057 & 0.109 \\
\hline \multicolumn{4}{|c|}{ Non-smooth, Beta } \\
\hline 100 & 0.005 & 0.042 & 0.100 \\
\hline 200 & 0.021 & 0.074 & 0.118 \\
\hline 400 & 0.018 & 0.062 & 0.124 \\
\hline 800 & 0.019 & 0.064 & 0.108 \\
\hline \multicolumn{4}{|c|}{ Smooth, Beta } \\
\hline 100 & 0.011 & 0.057 & 0.104 \\
\hline 200 & 0.015 & 0.059 & 0.121 \\
\hline 400 & 0.020 & 0.070 & 0.127 \\
\hline 800 & 0.018 & 0.060 & 0.112 \\
\hline
\end{tabular}

For the second the errors are drawn from a heavy-tail mixture of two $t$-distributions, one having a mode at 2 and the other at -2 , both having 5 degrees of freedom, with the same mixing probabilities as for the Gaussian mixture. For the third the errors are drawn from the Beta distribution with shape parameters $s_{1}=1+\delta 5\left(x_{1}+x_{2}\right)$ and $s_{2}=11-\delta 5\left(x_{1}+x_{2}\right)$. Per above, these errors are then rescaled to have unconditional mean zero and unit variance.

Table 3 reveals a curious feature of the non-smooth test - in the presence of discrete predictors, the test displays extreme size distortions rendering it completely unsuited for practical application 
TABle 3. Size for the Non-smooth and Smooth Tests (Empirical Rejection Probabilities Under the Null, $\delta=0$, Multivariate Mixed Continuous and Discrete Predictor Setting).

\begin{tabular}{|c|c|c|c|}
\hline$n$ & $\alpha=0.01$ & $\alpha=0.0$ & $\alpha=0.10$ \\
\hline \multicolumn{4}{|c|}{ Non-smooth, Gaussian Mixture } \\
\hline 100 & 0.285 & 0.545 & 0.678 \\
\hline 200 & 0.625 & 0.836 & 0.918 \\
\hline 400 & 0.950 & 0.994 & 0.998 \\
\hline 800 & 1.000 & 1.000 & 1.000 \\
\hline \multicolumn{4}{|c|}{ Smooth, Gaussian Mixture } \\
\hline 100 & 0.009 & 0.046 & 0.090 \\
\hline 200 & 0.013 & 0.056 & 0.100 \\
\hline 400 & 0.015 & 0.058 & 0.116 \\
\hline 800 & 0.024 & 0.081 & 0.159 \\
\hline \multicolumn{4}{|c|}{ Non-smooth, Student- $t$ Mixture } \\
\hline 100 & 0.361 & 0.632 & 0.752 \\
\hline 200 & 0.773 & 0.933 & 0.974 \\
\hline 400 & 0.993 & 1.000 & 1.000 \\
\hline 800 & 1.000 & 1.000 & 1.000 \\
\hline \multicolumn{4}{|c|}{ Smooth, Student- $t$ Mixture } \\
\hline 100 & 0.014 & 0.054 & 0.096 \\
\hline 200 & 0.013 & 0.061 & 0.108 \\
\hline 400 & 0.014 & 0.063 & 0.125 \\
\hline 800 & 0.012 & 0.074 & 0.140 \\
\hline \multicolumn{4}{|c|}{ Non-smooth, Beta } \\
\hline 100 & 0.518 & 0.782 & 0.877 \\
\hline 200 & 0.951 & 0.991 & 0.998 \\
\hline 400 & 1.000 & 1.000 & 1.000 \\
\hline 800 & 1.000 & 1.000 & 1.000 \\
\hline \multicolumn{4}{|c|}{ Smooth, Beta } \\
\hline 100 & 0.006 & 0.027 & 0.074 \\
\hline 200 & 0.004 & 0.040 & 0.084 \\
\hline 400 & 0.012 & 0.051 & 0.124 \\
\hline 800 & 0.023 & 0.110 & 0.200 \\
\hline
\end{tabular}

(its empirical rejection probability under the null approaches 1 as $n$ increases for all conventional levels). However, the smooth version of the test appears to be reasonably sized. The former is perhaps not too surprising given the literature on discrete/discontinuous distributions (Conover 1972, Gleser 1985, Choulakian et al. 1994, Lockhart et al. 2007). Given the extreme size distortions present, we make no attempt at power comparisons. 


\section{Application}

Racine \& Li (2017) impose a location-scale quantile model structure on a novel nonparametric quantile estimator that is based on kernel smoothing of a parametric quantile function in a particular manner. A practitioner concerned with their imposition of the location-scale structure might wish to use a pre-test approach, proceeding with the location-scale model if it is deemed appropriate versus an alternative model that does not rely on the location-scale assumption otherwise. They present two illustrative applications, one in which the covariate is continuous and one in which it is discrete, so these illustrations will serve to highlight the potential application of the proposed procedure.

We first consider an Italian gross domestic product (GDP) growth panel for 21 regions covering the period 1951-1998 (millions of Lire, 1990=base). There are $n=1,008$ observations on 2 variables, 'year' and 'gdp', and 'year' is a discrete predictor and is treated as such in what follows. Next we consider Canadian cross-section wage data consisting of a random sample taken from the 1971 Canadian Census Public Use Tapes for male individuals having common education (grade 13). There are $n=205$ observations in total on two variables, 'logwage' (logarithm of the wages) and 'age', age being treated as a continuous predictor. We report the test statistics and their bootstrapped $P$-values in Table 4 based on $B=999$ bootstrap replications.

TABle 4. Application of the Proposed Test to the Italian GDP Dataset and to the Canadian Cross-Section Wage Dataset.

\begin{tabular}{lcc} 
Dataset & $T_{K S}$ & $P$-value \\
\hline Canadian Wage & 0.4637124 & 0.3413413 \\
Italian GDP & 1.174512 & $<2 e-16$ \\
\hline
\end{tabular}

Table 4 reveals that the location-scale presumption is inappropriate for the Italian GDP data, but is appropriate for the Canadian wage data (though it is possible, given the small sample size of $n=205$, that this is a Type II error). These results indicate that practitioners can use Racine \& Li's (2017) quantile approach for the latter but ought to exercise caution when applying it to the former, which is consistent with the findings presented in Racine \& Li (2017) which used instead the non-smooth testing approach of Einmahl \& Van Keilegom (2008). 


\section{Concluding Remarks}

Numerous tests have been proposed that rely on the non-smooth empirical distribution function for their implementation; see Einmahl \& Van Keilegom (2008), Birke et al. (2017) and Neumeyer et al. (2016) by way of illustration. We demonstrate how the use of smooth estimators of distribution functions rather than their non-smooth counterparts can deliver tests having superior power profiles, which ought to be particularly appealing for practitioners.

\section{REFERENCES}

Aitchison, J. \& Aitken, C. G. G. (1976), 'Multivariate binary discrimination by the kernel method', Biometrika 63, 413-420.

Akritas, M. \& Van Keilegom, I. (2001), 'Nonparametric estimation of the residual distribution', Scandinavian Journal of Statistics 28, 549-568.

Anderson, T. W. \& Darling, D. A. (1952), 'Asymptotic theory of certain "goodness of fit" criteria based on stochastic processes', The Annals of Mathematical Statistics 23(2), 193-212.

Billingsley, P. (1999), Convergence of Probability Measures, Wiley \& Sons.

Birke, M., Neumeyer, N. \& Volgushev, S. (2017), 'The independence process in conditional quantile location-scale models and an application to testing for monotonicity', Statistica Sinica 27, 1815-1839.

Bowman, A. W., Hall, P. \& Prvan, T. (1998), 'Bandwidth selection for the smoothing of distribution functions', Biometrika 85, 799-808.

Choulakian, V., Lockhart, R. A. \& Stephens, M. A. (1994), 'Cramer-von Mises statistics for discrete distributions', The Canadian Journal of Statistics 22(1), 125-137.

Conover, W. J. (1972), 'A Kolmogorov goodness-of-fit test for discontinuous distributions', Journal of the American Statistical Association 67(339), 591-596.

Conover, W. J. (1999), Practical Nonparametric Statistics, third edn, Wiley.

Cramér, H. (1928), 'On the composition of elementary errors', Scandinavian Actuarial Journal 1, 13-74.

Davidson, R. \& MacKinnon, J. G. (2000), 'Bootstrap tests: How many bootstraps?', Econometric Reviews 19, 55-68.

Dette, H., Neumeyer, N. \& Van Keilegom, I. (2007), 'A new test for the parametric form of the variance function in non-parametric regression', Journal of the Royal Statistical Society: Series B 69, 903-917.

Einmahl, J. H. \& Van Keilegom, I. (2008), 'Specification tests in nonparametric regression', Journal of Econometrics 143, 88-102.

Escanciano, J. C., Pardo-Fernández, J. C. \& Van Keilegom, I. (2018), 'Asymptotic distribution free tests for semiparametric regressions with dependent data', Annals of Statistics 46, 1167-1196. 
Escanciano, J. \& Jacho-Chávez, D. T. (2012), ' $\sqrt{n}$-uniformly consistent density estimation in nonparametric regression models', Journal of Econometrics 12, 305-361.

Fan, J. \& Gijbels, I. (1996), Local Polynomial Modelling and Its Applications, Chapman \& Hall.

Gijbels, I., Van Keilegom, I. \& Zhao, Y. (2018), Gaussian copulas adjusted for nonparametric regression, Working paper, KU Leuven.

Gleser, L. J. (1985), 'Exact power of goodness-of-fit tests of Kolmogorov type for discontinuous distributions', Journal of the American Statistical Association 80(392), 954-958.

Jones, M. (1990), 'The performance of kernel density functions in kernel distribution function estimation', Statistics G Probability Letters $\mathbf{9}(2), 129-132$.

Kolmogorov, A. (1933), 'Sulla determinazione empirica di una legge di distributione', Giornale dell'Istituto Italiano degli Attuari 4, 1-11.

Li, C., Li, H. \& Racine, J. S. (2017), 'Cross-validated mixed-datatype bandwidth selection for nonparametric cumulative distribution/survivor functions', Econometric Reviews 36(6-9), 970-987.

Li, Q. \& Racine, J. S. (2004), 'Cross-validated local linear nonparametric regression', Statistica Sinica 14, 485-512.

Lockhart, R., Spinelli, J. \& Stephens, M. (2007), 'Cramér-von Mises statistics for discrete distributions with unknown parameters', Canadian Journal of Statistics 35(1), 125-133.

Neumeyer, N. (2008), 'A bootstrap version of the residual-based smooth empirical distribution function', Journal of Nonparametric Statistics 20(2), 153-174.

Neumeyer, N. (2009), 'Smooth residual bootstrap for empirical processes of non-parametric regression residuals', Scandinavian Journal of Statistics 36, 204-228.

Neumeyer, N., Noh, H. \& Van Keilegom, I. (2016), 'Heteroscedastic semiparametric transformation models: estimation and testing for validity', Statistica Sinica 26, 925-954.

Neumeyer, N. \& Van Keilegom, I. (2010), 'Estimating the error distribution in nonparametric multiple regression with applications to model testing', Journal of Multivariate Analysis 101, 1067-1078.

Neumeyer, N. \& Van Keilegom, I. (2018), 'Bootstrap of residual processes in regression: to smooth or not to smooth?', Biometrika (to appear).

Pardo-Fernández, J. C., Van Keilegom, I. \& González-Manteiga, W. (2007), 'Testing for the equality of $k$ regression curves', Statistica Sinica 17, 1115-1137.

Qiu, D. (2014), snpar: Supplementary Non-parametric Statistics Methods. R package version 1.0.

URL: https://CRAN.R-project.org/package=snpar

Racine, J. S. \& Li, K. (2017), 'Nonparametric conditional quantile estimation: A locally weighted quantile kernel approach', Journal of Econometrics 201(1), 72-94.

Ruppert, D. \& Wand, M. P. (1994), 'Multivariate locally weighted least squares regression', Annals of Statistics 22, 1346-1370. 
Smirnov, N. (1948), 'Table for estimating the goodness of fit of empirical distributions', Annals of Mathematical Statistics 19, 279-281.

Stute, W. (1982), 'The oscillation behavior of empirical processes', The Annals of Probability 10(1), 86-107.

Van der Vaart, A. W. \& Wellner, J. A. (1996), Weak Convergence and Empirical Processes, Springer.

Van Keilegom, I., González-Manteiga, W. \& Sánchez-Sellero, C. (2008), 'Goodness-of-fit tests in parametric regression based on the estimation of the error distribution', TEST 17, 401-415.

von Mises, R. E. (1928), Wahrscheinlichkeit, Statistik und Wahrheit, Julius Springer.

Wang, J., Cheng, F. \& Yang, L. (2013), 'Smooth simultaneous confidence bands for cumulative distribution functions', Journal of Nonparametric Statistics 25, 395-407. 
FACULTY OF ECONOMICS AND BUSINESS Naamsestraat 69 bus 3500

3000 LEUVEN, BELGIË

tel. + 3216326612

fax + 3216326791

info@econ.kuleuven.be www.econ.kuleuven.be 